University of Wollongong

Research Online

Faculty of Commerce - Papers (Archive)

Faculty of Business and Law

$1-1-2005$

\title{
The H-ternary line code power spectral density modelling investigation
}

Abdullatif Glass

Technical Studies Institute, UAE

Nidhal Abdulaziz

University of Wollongong in Dubai, nidhal@uow.edu.au

Eesa Bastaki

Dubai Silicon Oasis

Follow this and additional works at: https://ro.uow.edu.au/commpapers

Part of the Business Commons, and the Social and Behavioral Sciences Commons

\section{Recommended Citation}

Glass, Abdullatif; Abdulaziz, Nidhal; and Bastaki, Eesa: The H-ternary line code power spectral density modelling investigation 2005, 72-77.

https://ro.uow.edu.au/commpapers/2381

Research Online is the open access institutional repository for the University of Wollongong. For further information contact the UOW Library: research-pubs@uow.edu.au 


\title{
The $\mathrm{H}$-ternary line code power spectral density modelling investigation
}

\author{
Abstract \\ The power spectral density of the H-ternary line code is investigated in this paper. Accurate simulation \\ results are extracted from a simulation model that generates and encodes very long P-N binary sequence. \\ The simulation results are compared with that of the theoretical results obtained from the analytical \\ model. Both results almost fit with each other except for very low frequency components where the \\ simulation results drift slightly. This deviation is a result of the fact that the simulation model is able to \\ generate more realistic data sequence that mimics real-world data.

\section{Keywords} \\ Ternary, Line, Code, Power, Spectral, Density, Modelling, Investigation \\ Disciplines \\ Business | Social and Behavioral Sciences

\section{Publication Details} \\ Abdulaziz, N., Glass, A. \& Bastaki, E. (2005). The H-ternary line code power spectral density modelling \\ investigation. In B. Yeo, M. Gagnaire \& C. Omidyar (Eds.), Second IFIP International Conference on \\ Wireless and Optical Communications Networks (pp. 72-77). Dubai: IEEE.
}




\title{
The H-Ternary Line Code Power Spectral Density Modelling Investigation
}

\author{
Abdullatif Glass \\ Department of Planning and Training \\ Technical Studies Institute \\ P O Box 833 Abu Dhabi UAE \\ Tel: $97 \quad 545975$ Fax: 9758548 \\ e mail: aglass@ieee org
}

\author{
Nidhal Abdulaziz \\ College of Information Technology \\ University of Wollongong in Dubai \\ P O Box 83 Dubai UAE \\ Tel: $97 \quad 439 \quad 4$ Fax: $97 \quad 434585$ \\ e mail: NidhalAbdulaziz@uowdubai ac ae
}

\author{
Eesa Bastaki \\ Department of Education Trainning Research and Development \\ Dubai Silicon Oasis \\ P O Box 49 Dubai UAE \\ Tel: 974774 ; Fax: 974994 \\ e mail: eesa@dso ae
}

\begin{abstract}
The power spectral density of the H-ternary line code is investigated in this paper. Accurate simulation results are extracted from a simulation model that generates and encodes very long $P$ - $N$ binary sequence. The simulation results are compared with that of the theoretical results obtained from the analytical model. Both results almost fit with each other except for very low frequency components where the simulation results drift slightly. This deviation is a result of the fact that the simulation model is able to generate more realistic data sequence that mimics real-world data.
\end{abstract}

Keywords- Line codes Data transmission Power spectral density Telecommunication networks Simulation and Modelling

\section{INTRODUCTION}

Coding is the technique that is used in many telecommunication applications for the purpose of data transmission and storage The encoding principle is based on either adding or removing redundant bits to from the original binary data A variant form of coding to restructure the original binary pulse shape is also available The latter is mainly used in applications where transmission or storage of data are required The restructuring process is needed for the purpose of matching the encoded signal to the transmission storage media

Line codes have many desirable properties that make them attractive for certain applications These desirable features include small power spectral density bandwidth with no dc content frequent changes in signal level and hence adequate time information content for clock recovery and hence ease of transmitter receiver combination synchronisation error detection and correction capability and finally easy encoding and decoding methods and hence simple hardware implementation and cost effectiveness

Line coding has a generic form that is a multi level signal code Special cases can be obtained for binary ternary and quaternary line codes For binary ternary and quaternary codes; two three and four levels are used respectively to represent the code signal

Current applications of line codes are enormous in data transmission networks and in recording and storage of information systems The applications include local and wide area networks both wireless and wire connected and the new technology of the digital subscriber loops networks Further information about line codes classifications properties and their applications can be sought from [ 4]

In this paper a simulation model for the $\mathrm{H}$ ternary line is given and compared with the analytical model The next section provides a description of the procedure for how a binary sequence is encoded to an $\mathrm{H}$ ternary line code and then decoded back to its original state A review of the mathematical model of the power spectral density (PSD of the new line code together with its counterparts is given in section three Section four is devoted to a description of the simulation model and how it has been achieved Section five gives a discussion of the results and a comparison between mathematical and simulation models In the final section the conclusions are given

\section{H TERNARY LINE CODE OPERATION}

The $\mathrm{H}$ ternary line code operates on a hybrid principle that combines the binary NRZ L the ternary dicode and the polar RZ codes and thus it is called hybrid ternary The $\mathrm{H}$ ternary code has three output 
levels for signal representation; these are positive ( zero ( and negative ( The following subsections give a description of the procedures for the encoding and decoding principles

\section{A nco rOp raton}

The states shown in Table I depict the encoding procedure The $\mathrm{H}$ ternary code has three output levels for signal representation; these are positive ( zero ( and negative ( These three levels are represented by three states The state of the line code could be in any of these three states A transition takes place to the next state as a result of a binary input or and the encoder output present state The encoding procedure is as follows:

( The encoder produces level when the input is a binary and whether the encoder output present state is at or - level

( The encoder produces - level when the input is a binary and whether the encoder output present state is at or level

(3) The encoder produces level when the input is binary and the encoder present state is level or when the input is binary and the encoder present state is - level

(4 Initially the encoder output present state is assumed at level when the first binary bit arrives at the encoder input

TABLE I

ENCODER OPERATION PRINCIPLES

\begin{tabular}{|l|c|c|}
\hline \multirow{2}{*}{ Input Binary } & Output & \multicolumn{2}{c|}{ Ternary } \\
\cline { 2 - 3 } & Pr s nt tat & N tat \\
\hline & & \\
\hline & & \\
\hline & & \\
\hline & & \\
\hline & & \\
\hline & & \\
\hline
\end{tabular}

The operation procedure gives the reader sufficient information about the operation of this new line code scheme Further details and comparison together with design and modelling of the encoder can be sought from [4 57 9] The variation of this new line code is that it violates the encoding rule of NRZ L and dicode when a sequence of $\mathrm{s}$ or $\mathrm{s}$ arrives $\mathrm{In}$ the latter case it operates on the same encoding rule of polar RZ but with full period pulse occupancy

\section{$B D$ co r Op rat on}

Table II shows the input states of the $\mathrm{H}$ ternary decoder and its decoding procedure for an output binary It is a reverse process of the encoding operation given in the previous subsection The decoder has only two output states (binary whereas the input is three states (ternary The decoding procedure is as follows [ 9]

( The decoder produces an output binary when the input ternary is at level and whether the decoder output present state is a binary or

( The decoder also produces an output binary when the input ternary is at level and the decoder output present state is at a binary

(3 Similarly the decoder produces an output binary when the input ternary is at - level and whether the decoder output present state is a binary or

(4 Finally the decoder produces an output binary when the input ternary is at level and the decoder output present state is a binary

It is clear that the decoding process at the receiver is quite similar to that of the NRZ L code when the and levels are received The difference arises when level is received In which case the decision is made depending on the decoder output present state

TABLE II

DECODER OPERATION PRINCIPLES

\begin{tabular}{|l|c|c|}
\hline Input Ternary & \multicolumn{2}{|c|}{ Output } \\
& Pr s nt tat & N xt tat \\
\hline & & \\
\hline & & \\
\hline & & \\
\hline & & \\
\hline & & \\
\hline & & \\
\hline & & \\
\hline
\end{tabular}

\section{MATHEMATICAL MODELS}

The power spectral density of a line code is a very crucial factor in determining the bandwidth needed for the transmission of the encoded signal It also gives indication as how much the line code is able to compress the original binary code sequence bandwidth The following two sub sections give a brief analysis of the mathematical computation of $\mathrm{H}$ ternary line code and other peer codes and verification for the derived formula of the $\mathrm{H}$ ternary line code at zero frequency

A $R v$ wo Mat at ca Ana ys

The power spectral density (PSD of a line code can be evaluated using either deterministic or stochastic analysis techniques Since in our case the input data sequence is random the second approach is therefore adopted

The general expression of the PSD of a digital signal is given by [ 8 ]

$$
P_{c}\left(=\frac{\mid(\mid}{T} \sum_{k=-\infty}^{\infty} R(k \quad j \pi k T\right.
$$

where ( is the Fourier transform of the line code pulse shape $s(t$ of amplitude $A$ and duration $T$ and 
$R(k$ is its autocorrelation function It is evident that the above equation shows that the spectrum of the digital signal depends on two things: the pulse shape used and the statistical properties of the encoded signal Equation ( can also be rewritten in a simpler series form as follows

$$
P_{c}\left(=\frac{\mid(||}{T}\left[R+\sum_{k=}^{\infty} R(k \quad o s(\pi k T)]\right.\right.
$$

The pulse shape of the $\mathrm{H}$ ternary line code is a pulse of un $t$ amplitude with a duration $T$ The Fourier transform of the $\mathrm{H}$ ternary pulse is given by [8 ]

$$
\left(=T \frac{\sin T}{T}=T \sin c(T\right.
$$

The above $s n c$ function has a spectrum that extends to infinity for both the positive and negative frequencies

The statistical properties of the data are referenced to the autocorrelation function of the line code that is given by

$$
R\left(k=\sum_{=}\left(A A_{+k} P\right.\right.
$$

where $A$ and $A \quad{ }_{k}$ are the signal levels that correspond to the $t$ and $k t$ symbol positions that represent the $\mathrm{H}$ ternary line code respectively and $P$ is the probability of having the th $A$ and $A{ }_{k}$ product

To calculate the autocorrelation function of the line code for a different combination of symbols we first calculate the $R($ This means the autocorrelation function of the line code pulse symbol with itself From [7] the probability of occurrence of each symbol of the $\mathrm{H}$ ternary line code is equal This means the probability of the three transmitted code levels are equal i e $P=P=P=1 / 3 \quad$ Substituting these values together with their respected unit amplitude symbols into equation (4 for $N$ signal symbols and averaging the same signal symbols results in

$$
R\left(=\frac{N}{N}\left[\frac { N } { 3 } \left(+\quad+\frac{N}{3}\left(-\quad+\frac{N}{3}(\quad]=\frac{1}{3}\right.\right.\right.\right.
$$

The calculation of $R(k$ for all other values of $k$ excluding $k=$ can be determined using a tabulation method [89] The values of $R\left(\begin{array}{llll}R( & R(3 & \ldots & R(k\end{array}\right.$ can be found using the probabilities of all possible states The probabilities of each case also depend on the number of $\mathrm{H}$ ternary symbols that are considered For example the probabilities of each symbol are $P=1 / 4$ $P=1 / 8 \quad P_{3}=6 \quad$ and so on The autocorrelation function for each case using equation $(4$ is thus $R(=1 / 2 R(=1 / 4 R(3=1 / 8$ and so on for other values of $R_{k}$ The overall autocorrelation function for all values of $k$ excluding $k=$ is thus given as follows in a series form [8]

$$
R\left(k=\left(\frac{-^{k}}{k+}\right)=\left(-\frac{-}{}\right)^{k}\right.
$$

Substituting equations (5 and ( together with (3 into ( gives the PSD of the $\mathrm{H}$ ternary line code that is [8]

$$
P_{c}\left(=A T \sin c\left(T \left[\overline{3}+\sum_{k=}^{\infty}(-)^{k} \quad \text { os }(\pi k T]\right.\right.\right.
$$

The PSD of the $\mathrm{H}$ ternary line code is a re shaped form of the Fourier transform of a rectangular pulse having $A$ amplitude and duration of $T$ The re shaping function is the autocorrelation function of the $\mathrm{H}$ ternary line code

The normalised PSD results of the above derived formula for the $\mathrm{H}$ ternary code versus the normalised frequency is shown in figure Figure clearly shows that the bulk of the high weight frequency components of this line code are centred at 44 of the normalised frequency (signalling rate The spectrum also shows the line code has no dc component

To compare the $\mathrm{H}$ ternary line code spectrum with other line codes spectra under consideration the derived spectra formulae are given [ 8 ]

( Polar Non Return to Zero (NRZ L

$$
\begin{aligned}
& P_{c}(=A T \sin c(T \\
& P_{c}(=A T \sin c(T \sin (\pi T
\end{aligned}
$$

(3) Manchester

$$
P_{c}(=A T \sin c(T \quad \sin (\pi T
$$

Figure also shows the PSD comparison between the different line codes The figure clearly shows that the PSD of the H ternary line code overperforms NRZ $\mathrm{L}$ and lies between AMI and Manchester line codes spectra

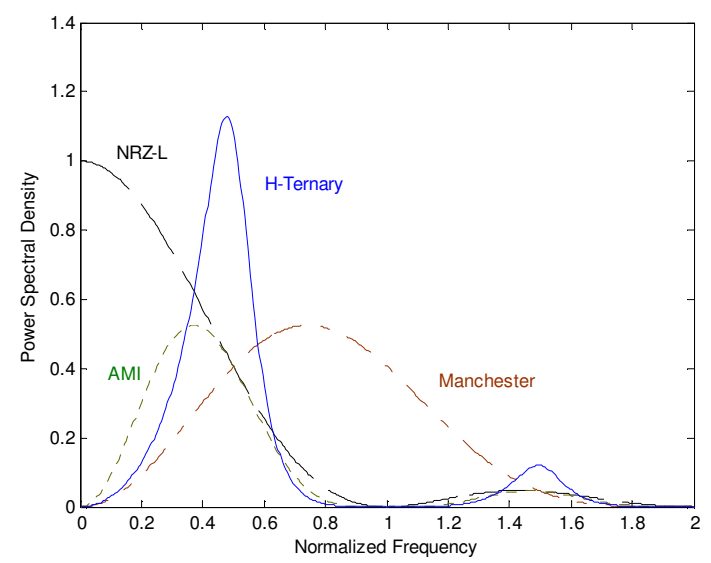

Fig Power spectra of different line codes

$B$ Inv st gatono $t$ H T rnary o Ana yt ca Mo

The mathematical PSD model derived in the previous sub section for the $\mathrm{H}$ ternary line code will be further investigated analytically in this sub section to validate its accuracy The reason for doing this is to 
check its validity with respect to the simulation results obtained from the simulation model

We need to prove that at zero frequency ( = equation ( 7 produces a value of zero which means there is no dc component This requires that the summation of the parameter which represents $R(k$ for all values of $k$ except for $k=$ should converge to a value of $1 / 3$ The above parameter is the autocorrelation function of the line code that is derived in equation (

The summation of $R$ ( $k$ that appears in equation ( 7 can be rearranged in the form

$$
{ }_{k}=\sum_{k=}^{\infty} r^{k}=\sum_{k=}^{\infty} r^{k}-
$$

where $r$ is equal to $1 / 2$

The summation ${ }_{k}$ of above geometric series is given by [ 3]

$$
{ }_{k}=\frac{}{-r}-=\frac{r}{-r}
$$

Substituting the value of $r=1 / 2$ into above equation yields

$$
k=\frac{-/}{-(-/}=-/ 3
$$

The bove value of $k$ represents the summation of $R(k$ given in equation 7 This gives a very important conclusion that the summation of $R(k$ tends to a value of $1 / 3$ for all values of $k$ except for $k=$ This in turn proves that the power spectral density given in the same equation has a zero value at zero frequency (dc

\section{SIMULATION MODEL}

The results of simulation are collected from a model written in Matlab The model generates $\mathrm{P} \mathrm{N}$ binary sequence of data which are then encoded into $\mathrm{H}$ ternary line code The encoded three level signal is then transformed to the frequency domain for the determination of the line code power spectral density using Matlab FFT routine The sampling frquency is chosen at four times the maximum rate of the $\mathrm{H}$ ternary encoded signal This was made to ensure the accuracy of the results of the spectra that were obtained from the simulation model

In the model the generated $\mathrm{P} \mathrm{N}$ binary sequence length is chosen to be bits These bits are encoded into three level $\mathrm{H}$ ternary symbols Figures and 3 show the simulation results for two different seeds The PSD results show relatively high weights of low frequency components This is slightly deviated from that of the analytical results

To improve the results and remove the discripency long $\mathrm{P} \mathrm{N}$ sequences are used It is expected that the drift in the results of the simulation model will be eliminated when the simulation model is run for long sequences Thus the simulation model has been modified to generate long $\mathrm{P} \mathrm{N}$ sequence of one million binary bits As before the new sequence is encoded and transformed to the frequency domain and the results are collected Althought it took about times the time needed for the previous bit model to finish the run and gather the results the results came almost similar to that of the bit model Figure 4 shows the results for $\quad H$ ternary code symbols

It is also shown that different seeds give different weights for the different frequency components of the line code PSD as shown in figures and 3 To reduce the difference the simulation model has been run for

times for different random $\mathrm{P} N$ sequence seeds The results from these runs are then added up and averaged over the number of runs and displayed in figure 5

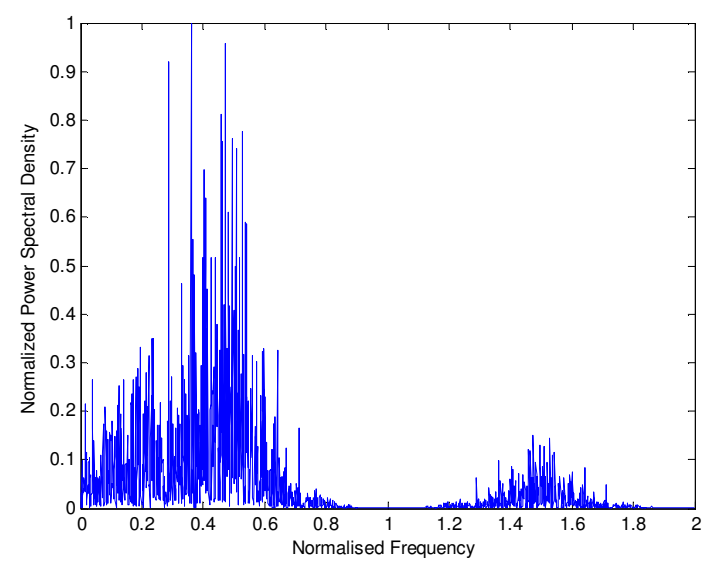

Fig Normalised power spectral density of the simulation results for a bit P N sequence (seed 345

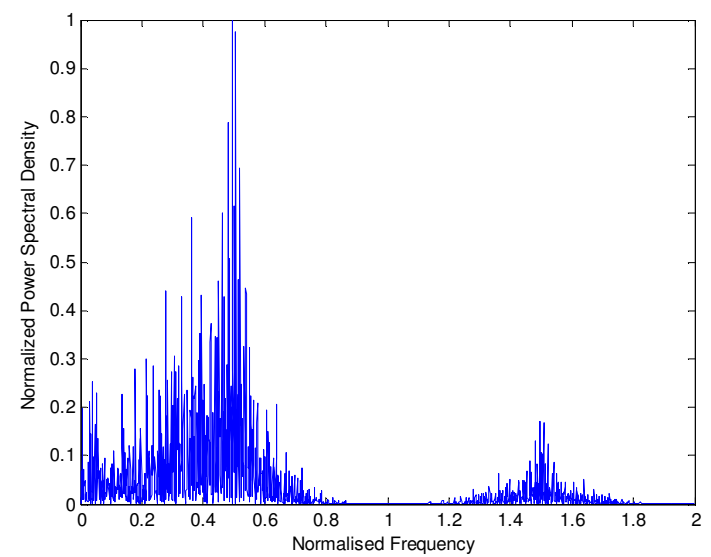

Fig 3 Normalised power spectral density of the simulation results for a bit $\mathrm{P} \mathrm{N}$ sequence (seed 543

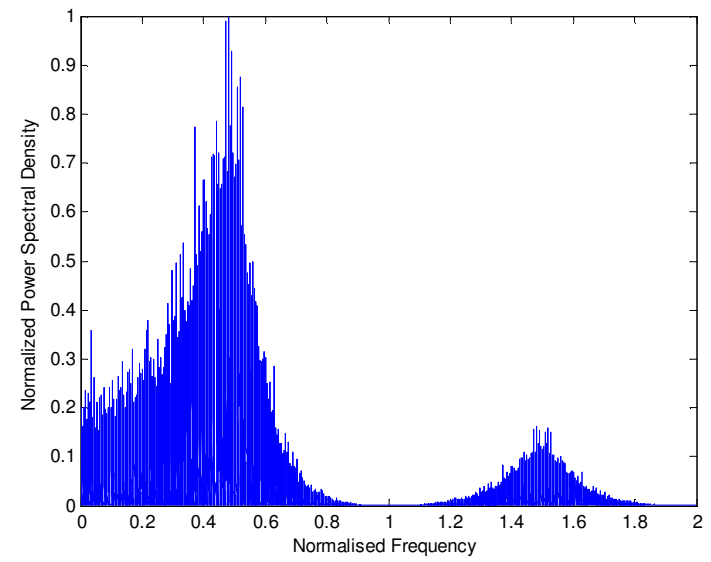

Fig 4 Normalised power spectral density of the simulation results for a bit $\mathrm{P} \mathrm{N}$ sequence (seed 345 


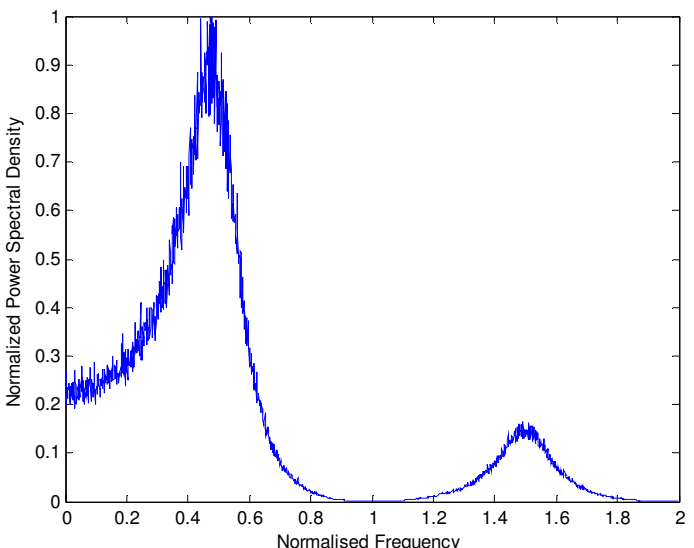

Fig 5 Normalised avearged power spectral density of the simulation results for different seeds

\section{RESULTS COMPARISON AND DISCUSSION}

The mathematical model of the $\mathrm{H}$ ternary line code and its results have been thoroughly investigated and proved percent accurate Comparing the simulation results obtained from the simulation model with that of the mathematical model can be seen in figure In this figure the analytical results are compared with the simulation results for only a single seed The results show a minor deviation from each other for both models at the low frequency components of the line code spectra It also shows that the simulation discrete line spectra are fluctuating above and below the solid line of the mathematical model Longer $\mathrm{P} N$ binary sequence is then suggested to improve the simulation results to match that of the analytical results at low frequency components The latter encoded sequence spectra however show almost the same results of that of the shorter sequences as shown in figure 4

In order to reduce the fluctuation in the simulation result spectra for the whole simulated spectrum several runs of the simulation model have been carried out for different $\mathrm{P} \mathrm{N}$ sequence seeds The results of these runs are then added up and averaged over the number of runs and are displayed in figure 7 together with that of the analytical model Figure 7 show excellent agreement of both models at all frequency spectra except for that of the lower frequency region

Figure 7 shows clearly that the analytical model starts from zero PSD value at zero frequency however this is not the case for the simulation results The analytical results are accurate however they are unable to exactly reflect the practical case of the line code sequence This does mean that the simulation model is able to generate binary sequence that is then encoded to a three level code signal which has higher weight of repeated patterns at low frequencies that are close to zero

The $\mathrm{H}$ ternary line code considered here is a modification of other predecessor codes that are used for base band and pass band data transmission The new code exploits the merits of these codes and eliminates their deficiencies The reshaping process of the $\mathrm{H}$ ternary pulses show preferred spectra with no dc component that enables better use of the allocated spectrum It provides a signalling rate of around 44 percent of the original data bit rate provides better timing information for encoder decoder synchronisation and many other desirable features

The $\mathrm{H}$ ternary line code has a noise performance which is superior to its predecessor line codes at low level signal to noise ratio [7] This superiority enables the code to operate at lower power and perform better than the other line codes

The code has also got the property of single error detection [5 9] A property that is very much desirable in line codes This came into effect due to the fact that encoding has a correlative relation between the adjacent line code symbols

The new line code hardware is relatively more complex in implementation of the encoder and decoder This complexity however meets a relative simplicity in the clock recovery circuit at the receiving end This is due to the fact that for every transmitted H ternary symbol there is a change in signal level

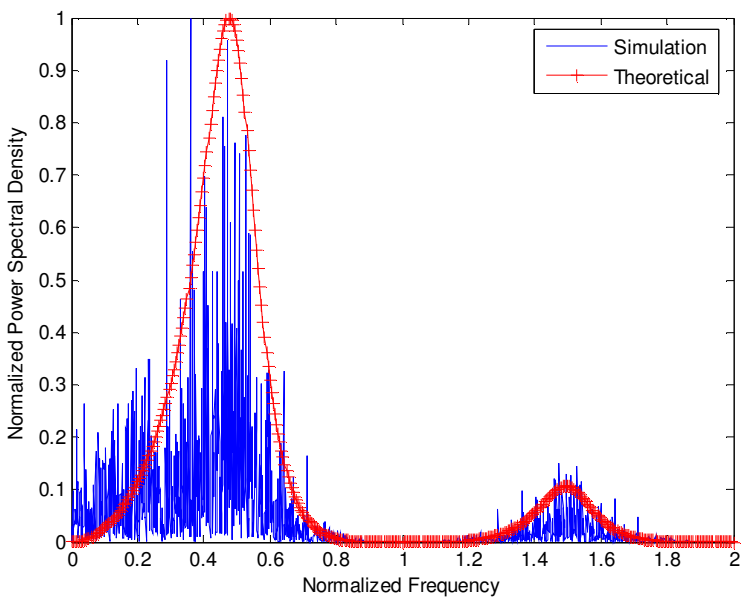

Fig Results comparison of the theoretical and the simulation models (single seed 345

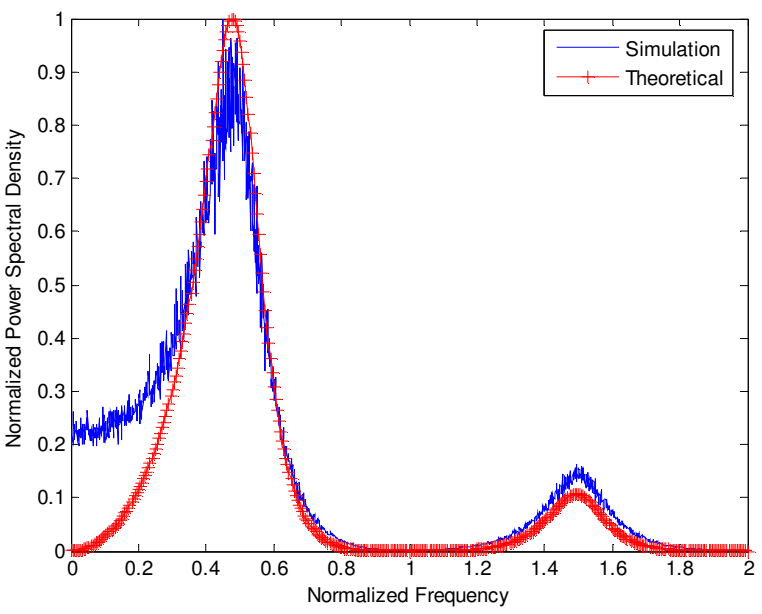

Fig 7 Results comparison of the theoretical and the simulation models ( $\quad$ seeds 


\section{CONCLUSIONS}

A comparison between two models of the $\mathrm{H}$ ternary line code is presented Investigation of the analytical model has been done together with the simulation model The results obtained from both models are correct and accurate The simulation results however reveal a fact that this model is able to generate sequence that are more realistic and closely resembles real world data The overall results however revealed an important fact that the PSD of this new line code has very small bandwidth that is centred at about 44 of that of the normalised signalling rate of the binary sequence with no dc component The code has many other desirable features such as timing information content and a single error detection capability

\section{REFERENCES}

[ ] W Cattermole "Principles of digital line coding" International J of Electronics vol 55 no pp 333983

[ ] F Xiong Digital modulation techniques Artech House pp 783

[3] $\mathrm{H}$ Dutton and $\mathrm{P}$ Lenhard High speed networking technology: an introductory survey Prentice Hall 995 pp 5

[4] A Glass and E Bastaki "H Ternary line code for data transmission "International Conference on Communications Computer and Power (ICCCP Sultan Qaboos University Muscat Oman 4 February pp 7
[5] A Glass B Ali and E Bastaki "Design and modelling of $\mathrm{H}$ ternary line encoder for digital data transmission" International Conference on Info Tech \& Info Net (ICII Beijing China Oct 9 Nov pp 5357 [ ] A Glass B Ali and N Abdulaziz "H Ternary line decoder for digital data transmission: circuit design and modelling" th International Symposium on Digital Signal Processing for Communication Systems (DSPCS

Sydney Australia Jan $83 \quad$ pp 4953

[7] A Glass E Bastaki and N Abdulaziz "Performance of $\mathrm{H}$ ternary line code in the presence of noise: analysis and comparison" $4^{\text {th }}$ Middle East Symposium on Simulation and Modelling (MESM American University of Sharjah Sharjah UAE Sept 383 pp $4 \quad 5$

[8] A Glass N Abdulaziz and E Bastaki "Mathematical analysis of the power spectral density of the $\mathrm{H}$ ternary line code" nd IEEE GCC Conference Manama Bahrain Nov $\begin{array}{lll}3 & 5 & 4 \mathrm{pp}\end{array}$

[9] A Glass N Abdulaziz and E Bastaki "The Power Spectral Density of the H Ternary Line Code: A Simulation Model and Comparison" Submitted for publication at the $3^{\text {rd }}$ IEEE International Conference on Information Technology and Applications (ICITA 5 UTS Sydney Australia July 4 $7 \quad 5$

[ ] P Lathi Modern digital and analog communication systems ( $3^{\text {rd }} \mathrm{Ed}$ Oxford University Press 988 pp 94353

[ ] L W Couch Digital and analog communication systems $\left(5^{\text {th }}\right.$ Ed Prentice Hall $997 \mathrm{pp} \quad 7 \quad 5$

[ ] S Haykin Digital communications John Wiley 988 pp $34 \quad 7$

[ 3] K Stroud Engineering mathematics $\left(4^{\text {th }}\right.$ Ed Macmillan 995 pp 53759 